\title{
Interpretation of Backlit Droplet Images from ISS Droplet Combustion Experiments
}

\author{
Fei $\mathrm{Yu}^{1}$ and Benjamin D. Shaw ${ }^{2}$ \\ ${ }^{1}$ Computer Science Department, University of California - Davis, Davis, CA; ${ }^{2}$ Mechanical and Aerospace \\ Engineering Department, University of California - Davis, Davis, CA
}

\begin{abstract}
Backlit droplet images are evaluated for droplet combustion experiments that have been performed on the International Space Station. The focus of the present analyses is on non-sooting or lightly-sooting droplets. The influences of diffraction, interference, and partial coherence on droplet images are considered via Fourier optics modeling. It is found that light diffraction at the droplet edge can contribute significantly to errors in droplet size measurements. Other error sources include background light variations and partial coherence effects. An image-processing algorithm is proposed to account for the effects of diffraction, partial coherence, and background light variations on measurements of droplet sizes.
\end{abstract}

\section{INTRODUCTION}

This research is in support of two experiments performed onboard the International Space Station (ISS), the Droplet Flame Extinguishment in

Key words: Droplet Combustion; Image Analysis; International Space Station; Partial Coherence

Correspondence to: Benjamin D. Shaw

University of California - Davis

Mechanical and Aerospace Department

2132 Bainer Hall

1 Shields Avenue

Davis, CA 95616

Telephone: 530-752-6801

E-mail: bdshaw@ucdavis.edu
Microgravity (FLEX), and the Fundamental Studies in Droplet Combustion and Flame Extinguishment in Microgravity (FLEX-2) experiments. The FLEX and FLEX-2 research efforts use reduced-gravity droplet combustion to investigate flammability and extinction under conditions relevant to space exploration, as well as fundamental aspects of condensed-phase combustion of fuels. The focus of the present research is to evaluate the optical characteristics of the backlit droplet images that are obtained from the experiments.

In both the FLEX and FLEX-2 experiments, droplets were burned within the Multiuser Droplet Combustion Apparatus (MDCA) (Deitrich et al., 2013). This facility allows for combustion of either free floating or fiber-supported (tethered) droplets inside a pressure chamber. Initial droplet diameters are generally in the range of $2-6 \mathrm{~mm}$. The gas composition and pressure inside the chamber are controlled and droplets of different initial compositions can be burned. The MDCA facility allows measurements of droplets and flames via imaging, soot volume fractions via laser extinction, radiation emitted from flames via radiometers, as well as chamber temperatures, pressures, and compositions.

\section{BACKLIT DROPLET IMAGES}

The images used for droplet sizing are produced by collimating the light from a fibercoupled light emitting diode (LED) and then directing this light past a droplet and into a telecentric lens. This lens directs the light into a 
monochrome digital camera (1024 x 1024 pixels). The collimated light and telecentric lens combination ideally produces well-defined shadows of objects on the camera image sensor array. Similar types of systems are used in machine vision applications (Webster, 1999; Senchenko and Chugui, 2011).

A representative image of a heptane droplet burned in the MDCA is shown in Figure 1a. Figure $1 \mathrm{~b}$ shows a pixel intensity profile along a line drawn through the droplet. This intensity profile displays several characteristics that are important for image processing. The background light intensity away from the droplet edge has small fluctuations and a sharp peak in pixel intensity is apparent at each edge of the droplet where the profile is taken. There is a small jump in pixel intensity near the droplet center. The background light intensity is appreciably different on either side of the droplet.
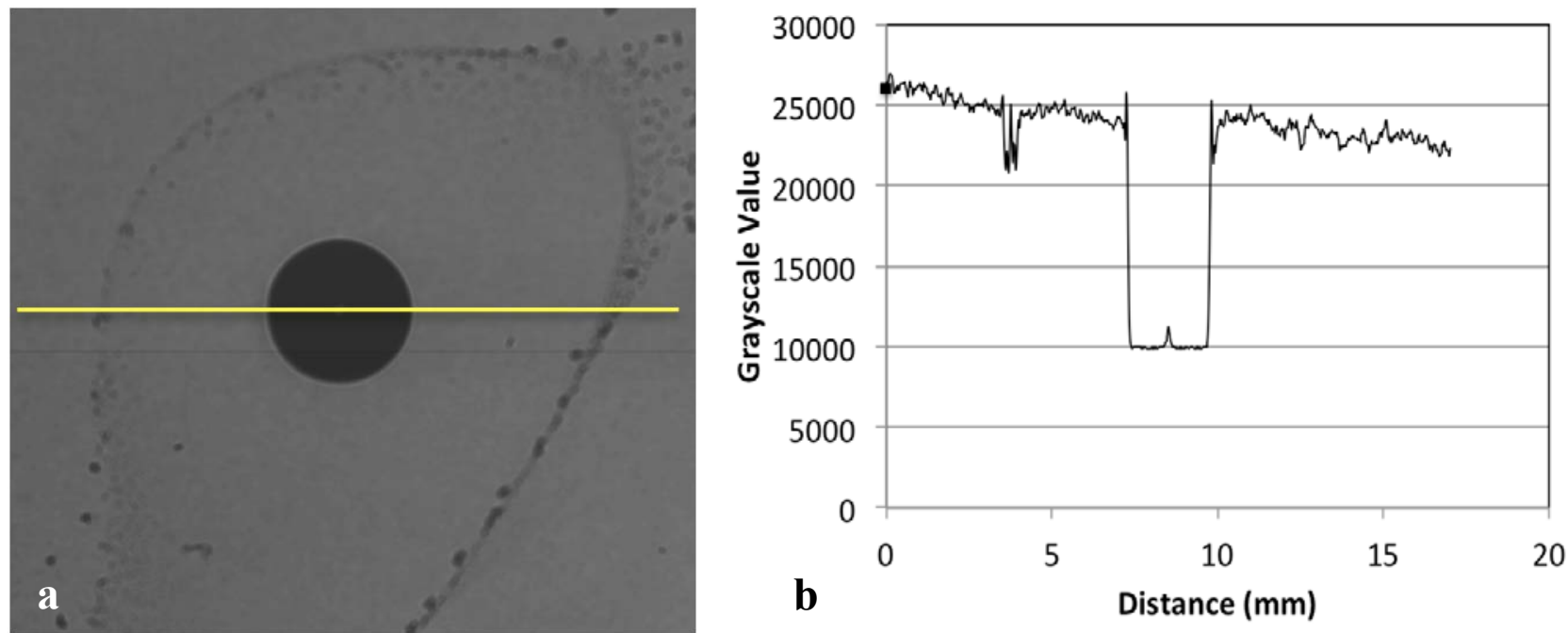

Figure 1. (a) Representative backlit droplet image; and (b) grayscale profile across the droplet and through the droplet center.

Figure 2a shows the same droplet as in Figure $1 \mathrm{a}$, but where the view is zoomed in to show the droplet edge. Shown in Figure $2 b$ is a normalized pixel intensity profile defined to be zero within the droplet interior and unity in the background away from the droplet edge. The intensity profile shows the structure of the peak, as well as the fact that the droplet edge is indistinct and is apparently smeared over a finite-width region. The smearing of the droplet edge is expected with a collimated backlight (Skinner, 1965; Senchenko and Chugui, 2011). The light beam will be diffracted at the droplet edge, causing light to enter the geometrical shadow zone of the droplet, as shown schematically in Figure 3. The appearance of the peak is expected, based on interference effects that will be present if the backlight is partially or fully coherent (Fowler and Litorja, 2003; Senchenko and Chugui, 2011). If the backlight is incoherent, then the peak is not expected, though the droplet edge will still be indistinct because of diffraction (Skinner, 1965).

As noted above, the backlight was produced by directing the light from an LED into an optical fiber and then into the collimation system. Directing light through an aperture, such as the entrance of an optical fiber, can introduce partial coherence, which is consistent with the appearance of the intensity peak. Since objects like steel and glass spheres and solid objects placed in the field of view of a functionally equivalent MDCA apparatus used for ground characterizations of the flight apparatus show the same edge structure (e.g., a local peak and a smeared edge), we can assume that the appearance of a peak is a result of diffraction of partially coherent light. For example, Figure 4 and Figure 5 show images and grayscale profiles for stainless steel and glass spheres. 

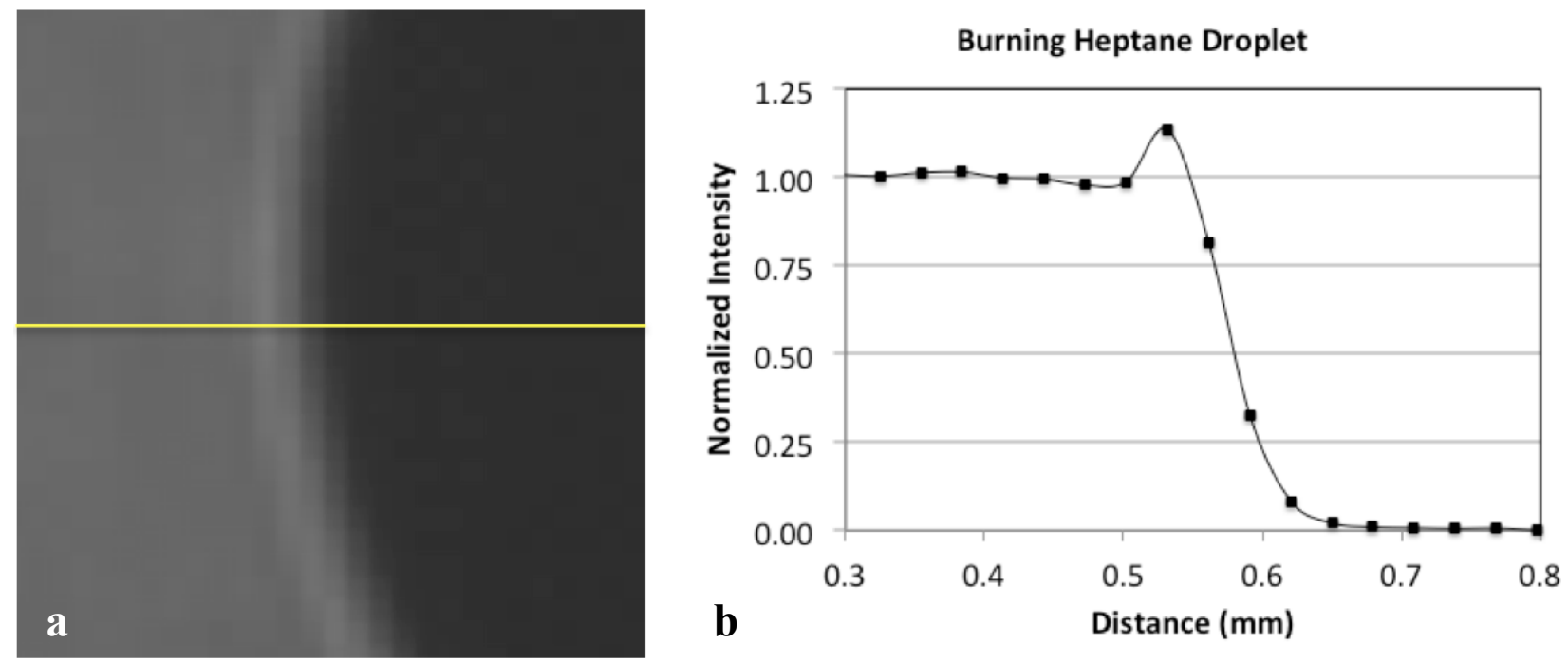

Figure 2. (a) Digitally-zoomed droplet image; and (b) normalized grayscale profile showing intensity variations across a droplet edge.

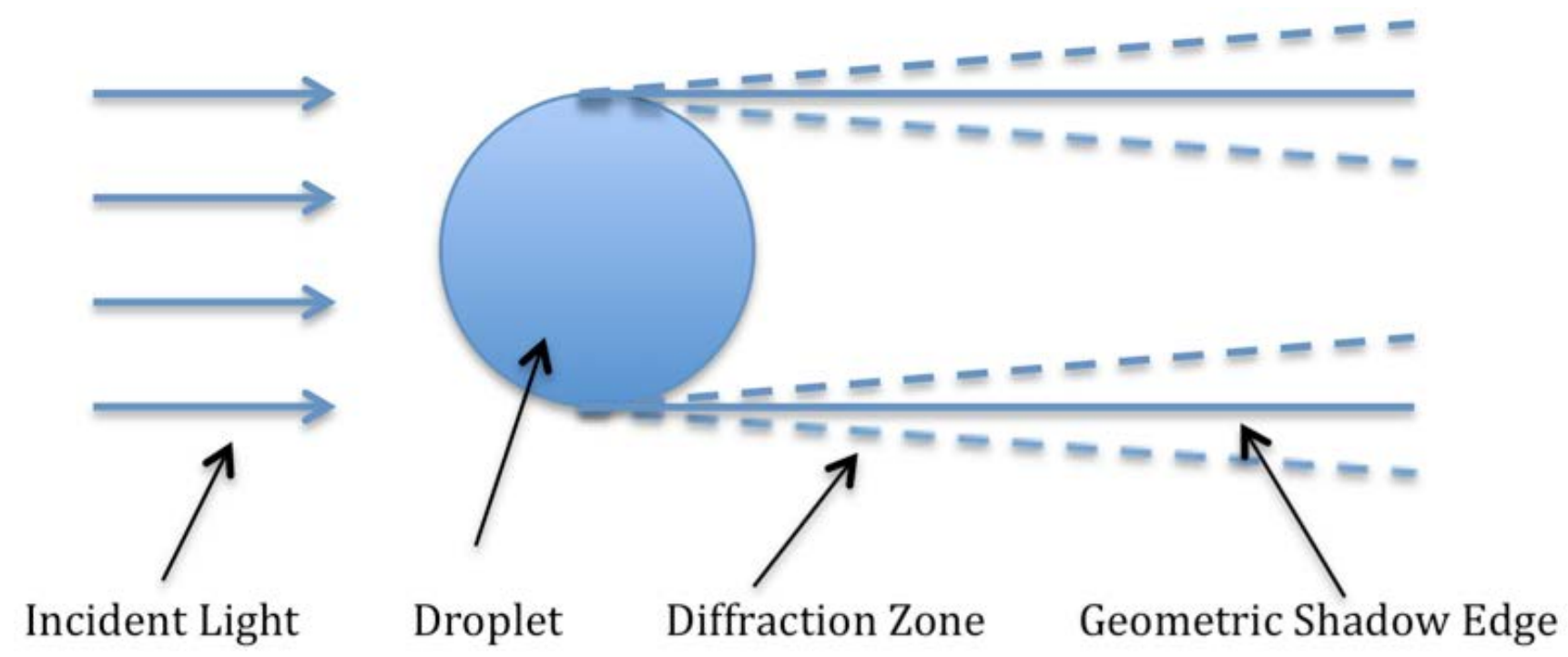

Figure 3. Schematic of diffraction of light by a droplet. 

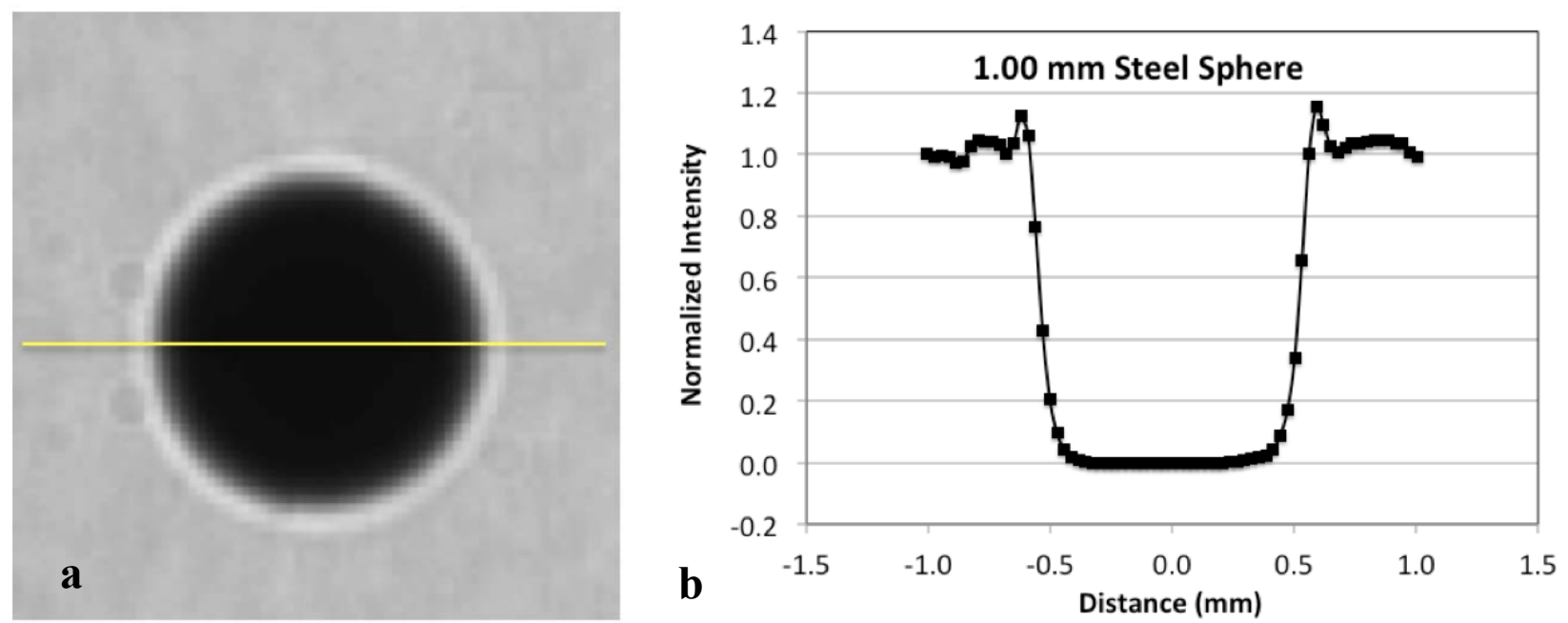

Figure 4. (a) Image of a stainless-steel sphere; and (b) normalized grayscale profile across the sphere.
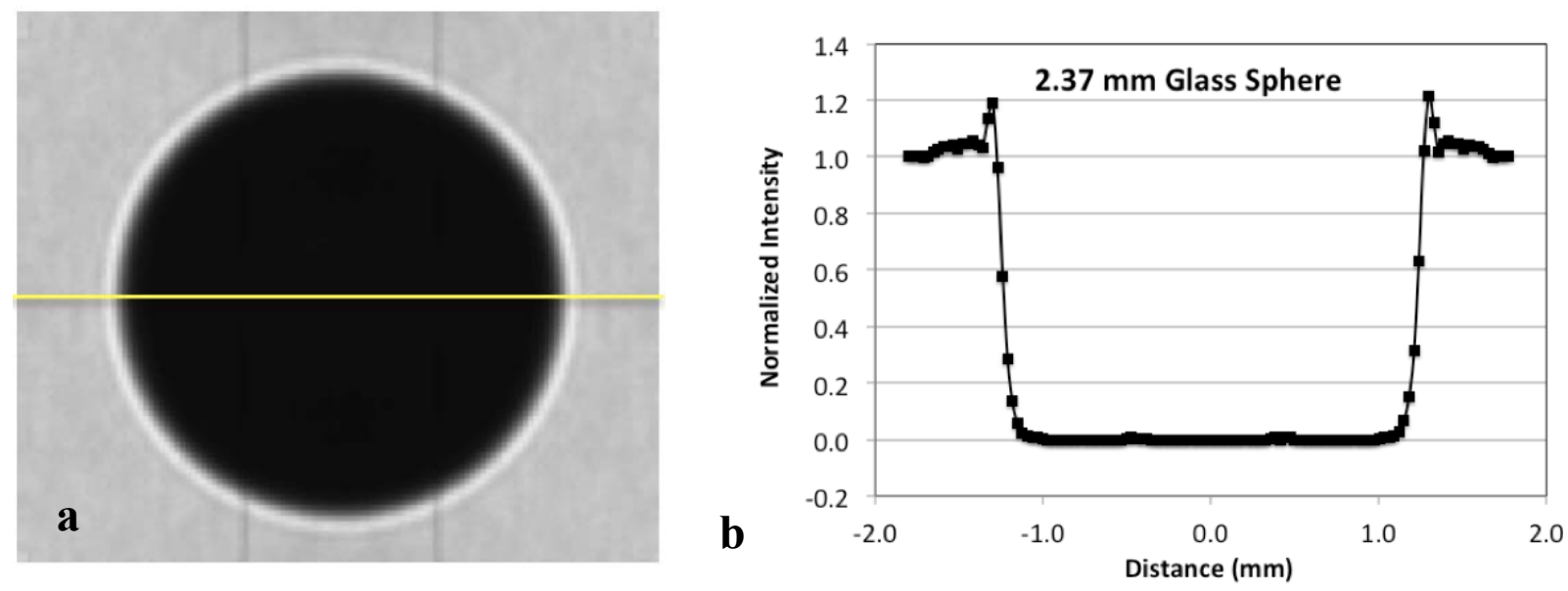

Figure 5. (a) Image of a glass sphere; and (b) normalized grayscale profile across the sphere.

The influences of partial coherence of the backlight were investigated theoretically by using computational Fourier optics models (Voelz, 2011). The numerical model was used to calculate the light intensity field at the image plane, assuming the only diffraction that occurs is from the edge of a droplet. The droplet was modeled as an opaque disk subject to the following types of collimated illumination: (1) completely incoherent; (2) completely coherent; and (3) partially coherent.

In the model, we consider the general case where a planar electromagnetic wave of wavelength $\lambda$ is incident on a planar surface (the aperture plane) that partially blocks the wave from reaching another planar surface (the image plane). The planar surfaces are parallel and separated by the distance $\mathrm{z}$ and the Cartesian coordinates $\mathrm{x}$ and $\mathrm{y}$ are parallel on each plane. We consider the following imaging situations: (1) completely coherent; (2) completely incoherent; (3) partially coherent; and (4) geometrical optics.

Suppose that $E_{1}$ and $E_{2}$ are the optical fields (proportional to the electric fields) at the aperture and image planes, respectively. In addition, we define $\mathrm{I}_{1}=\mathrm{E}_{1} \mathrm{E}_{1}{ }^{*}$ and $\mathrm{I}_{2}=\mathrm{E}_{2} \mathrm{E}_{2}{ }^{*}$ as the 
irradiances at the aperture and image planes, respectively, where $" * "$ represents complex conjugation. Because optical fields oscillate at very high frequencies, e.g., $10^{14} \mathrm{~Hz}$, imaging systems, such as cameras, respond to time averages of the magnitude of the optical fields, which are represented by the irradiances. As a result, we calculate irradiances at the image plane.

Geometrical Optics:

Completely Coherent:

Completely Incoherent:

Partially Coherent:
The impulse-response function (Voelz, 2011) is defined to be $\mathrm{h}=\mathrm{ze} \mathrm{i}^{\mathrm{i} \lambda \mathrm{r}} /\left(\mathrm{i} \lambda \mathrm{r}^{2}\right)$, where $\mathrm{r}=\left(\mathrm{x}^{2}+\mathrm{y}^{2}\right.$ $\left.+\mathrm{z}^{2}\right)^{1 / 2}$. This impulse-response function, which propagates the field from the aperture to the image plane, corresponds to the Rayleigh-Sommerfeld diffraction solution (Voelz, 2011). The image plane solutions are determined using spatial convolutions, which are denoted by $\otimes$. The specific forms of the solutions are as follows:

$$
\begin{aligned}
& E_{2}=E_{1}, I_{2}=I_{1} \\
& E_{2}=E_{1} \otimes h, I_{2}=E_{2} E_{2}{ }^{*} \\
& I_{2}=\left(h h^{*}\right) \otimes I_{1} \\
& E_{2}=\left(E_{1} T\right) \otimes h,<I_{2}>=<E_{2} E_{2}^{*}>
\end{aligned}
$$

center. The interface between the background and the droplet is sharp, as would be expected for geometrical optics. Figure 7 and Figure 8 show analogous information, but for the situation where the backlight is completely incoherent or completely coherent, respectively. In both cases, the interface between the droplet and the background is smeared over a finite region. The completely coherent case also shows "ringing" associated with diffraction rings, with a sharp peak near the droplet edge. In contrast, the completely incoherent case does not exhibit ringing or any peaks in intensity. It is notable that Figure $10 \mathrm{~b}$ has a peak in intensity at the droplet center. This peak is the Arago spot (Voelz, 2011).
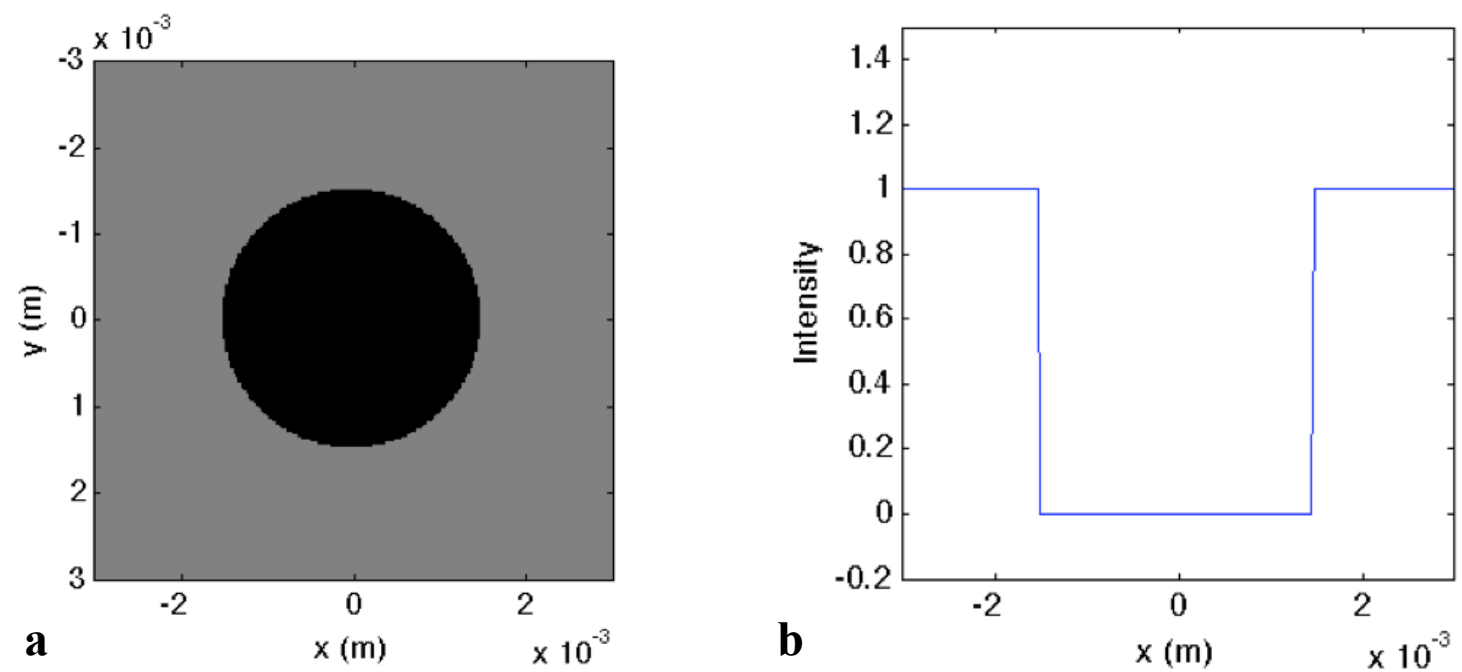

Figure 6. (a) Simulated image of a $3 \mathrm{~mm}$ droplet in the geometrical optics limit; and (b) the intensity profile along a line through the droplet center. 

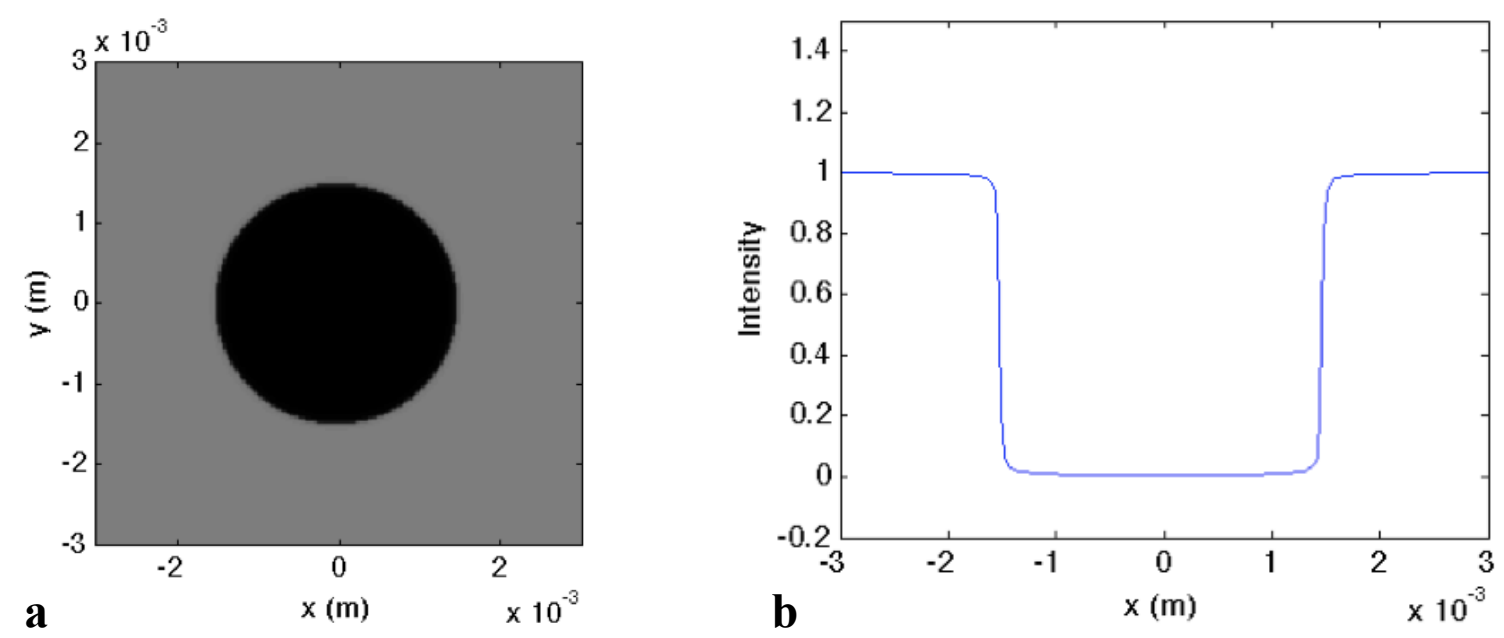

Figure 7. (a) Simulated image of a $3 \mathrm{~mm}$ droplet with completely coherent backlighting; and (b) the intensity profile along a line through the droplet center.
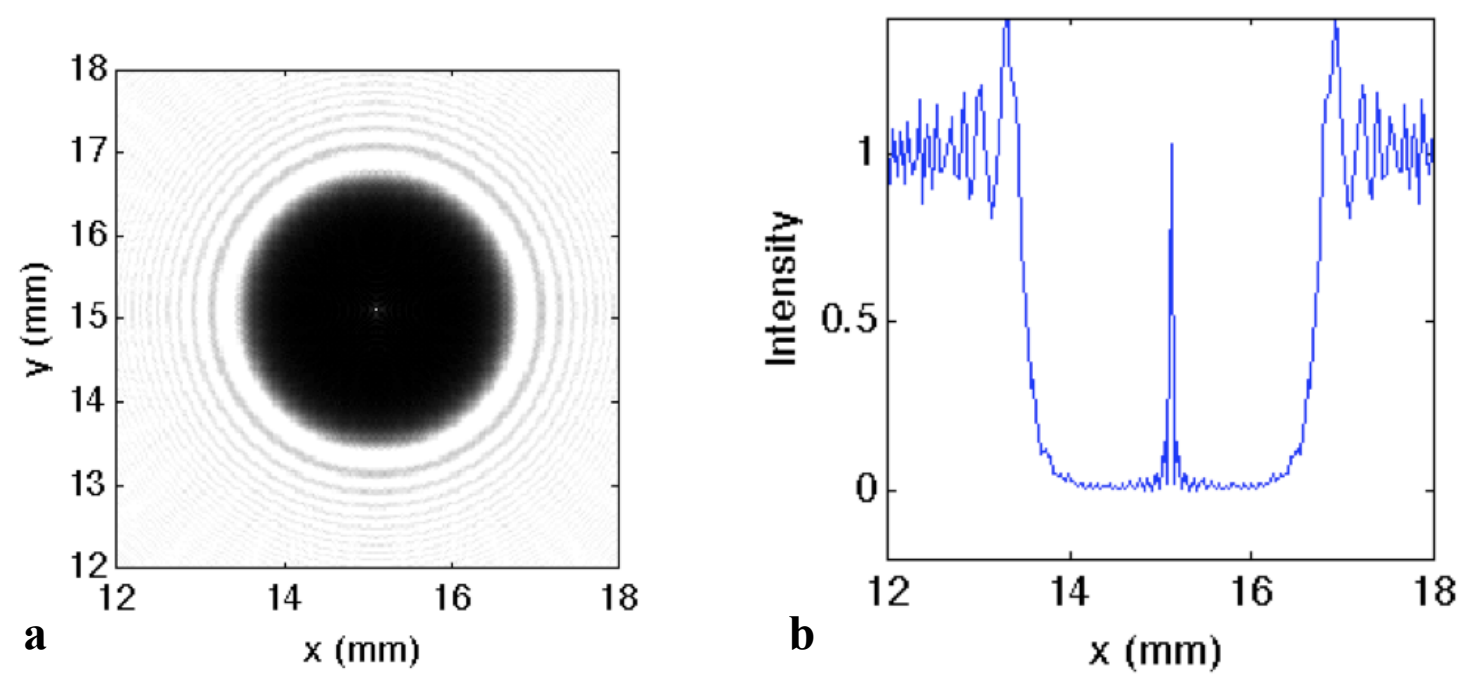

Figure 8. (a) Simulated image of a $3 \mathrm{~mm}$ droplet with completely incoherent backlighting; and (b) the intensity profile along a line through the droplet center.

Figure 9 shows results from an optical model that considered partial coherence via averaging the results from a large number of random transmittance screens (Voelz, 2011). The results in Figure 9a and Figure 9b show strong similarity to the experimental images, in that a sharp peak is evident near the droplet surface. As a result, it is likely the optical system used in these experiments was not fully incoherent or fully coherent, but was rather somewhere in between, i.e., it was partially coherent. This is important because locating the droplet edge in an image requires knowledge of the coherence level, as described below. 

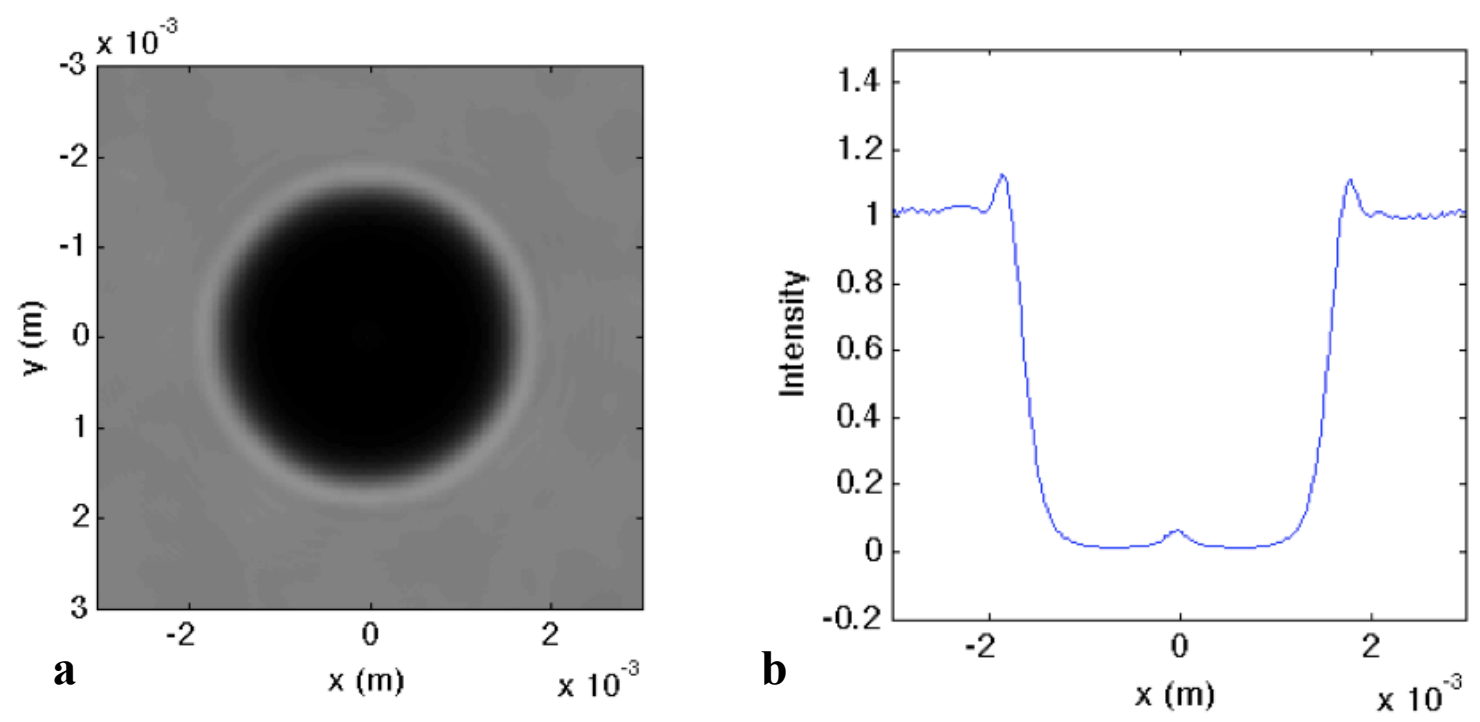

Figure 9. (a) Simulated image of a $3 \mathrm{~mm}$ droplet with partially coherent backlighting; and (b) the intensity profile along a line through the droplet center.

\section{EVALUATION OF DROPLET SIZES FROM IMAGES}

In this analysis we determine the edge coordinates at a number $(\mathrm{N})$ of points. A circle is then fit to these points and the diameter of this circle is taken to be the droplet diameter. This is the approach used by Dembia et al. (2012) for evaluating sizes of droplets that are strongly sooting, though Dembia et al. (2012) did not consider diffraction or partial coherence effects. For the circle fit procedure, we follow the analytical procedure of Kasa (1976). We provide some of the details of this analysis below because Kasa (1976) derived a best-fit equation for the radius $\mathrm{R}$ of a circle, while we are interested in a best-fit equation for $R^{2}$. This is because $R^{2}$ is needed for calculating burning rates.

Assume we have identified $\mathrm{N}$ points $\left(\mathrm{x}_{\mathrm{i}}, \mathrm{y}_{\mathrm{i}}\right)$ along the droplet edge in a Cartesian $(\mathrm{x}, \mathrm{y})$ coordinate system (Figure 10). To fit a circle we minimize the function

$$
\mathrm{u}=\sum_{\mathrm{i}=1}^{\mathrm{N}}\left[\left(\mathrm{x}_{\mathrm{i}}-\mathrm{A}\right)^{2}+\left(\mathrm{y}_{\mathrm{i}}-\mathrm{B}\right)^{2}-\mathrm{R}^{2}\right]^{2}
$$

by varying the parameters $A, B$, and $R^{2}$. The variables $\mathrm{A}$ and $\mathrm{B}$ correspond to the $\mathrm{x}$ and $\mathrm{y}$ values, respectively, at the center of the best-fit circle and $\mathrm{R}^{2}$ is the square of the radius of this circle. It is also noted that $r_{i}=\left[\left(x_{i}-A\right)^{2}+\left(y_{i}-B\right)^{2}\right]^{1 / 2}$ is the distance from the center of the best-fit circle to a data point. Setting

$$
\frac{\partial \mathrm{u}}{\partial \mathrm{A}}=\frac{\partial \mathrm{u}}{\partial \mathrm{B}}=\frac{\partial \mathrm{u}}{\partial \mathrm{R}^{2}}=0
$$

and defining $C=R^{2}-A^{2}-B^{2}$ yields a linear system of three equations for $A, B$, and $C$. If we define the matrices 


$$
\begin{gathered}
\mathrm{D}=\left[\begin{array}{ccc}
2 \sum \mathrm{x}_{\mathrm{i}} & 2 \sum \mathrm{y}_{\mathrm{i}} & \mathrm{N} \\
2 \sum \mathrm{x}_{\mathrm{i}}^{2} & 2 \sum \mathrm{x}_{\mathrm{i}} \mathrm{y}_{\mathrm{i}} & \sum \mathrm{x}_{\mathrm{i}} \\
2 \sum \mathrm{x}_{\mathrm{i}} \mathrm{y}_{\mathrm{i}} & 2 \sum \mathrm{y}_{\mathrm{i}}^{2} & \sum \mathrm{y}_{\mathrm{i}}
\end{array}\right] \\
\mathrm{E}=\left[\begin{array}{c}
\sum\left(\mathrm{x}_{\mathrm{i}}^{2}+\mathrm{y}_{\mathrm{i}}^{2}\right) \\
\sum\left(\mathrm{x}_{\mathrm{i}}^{3}+\mathrm{x}_{\mathrm{i}} \mathrm{y}_{\mathrm{i}}^{2}\right) \\
\sum\left(\mathrm{x}_{\mathrm{i}}^{2} \mathrm{y}_{\mathrm{i}}+\mathrm{y}_{\mathrm{i}}^{3}\right)
\end{array}\right] \\
\mathrm{Q}=\left[\begin{array}{c}
\mathrm{A} \\
\mathrm{B} \\
\mathrm{C}
\end{array}\right]
\end{gathered}
$$

then $\mathrm{DQ}=\mathrm{E}$ and the matrix $\mathrm{Q}$ can be obtained as follows.

$$
\mathrm{Q}=\mathrm{D}^{-1} \mathrm{E}
$$

The square of the droplet diameter, $\mathrm{d}$, is then calculated as

$$
d^{2}=4 R^{2}=4\left(A^{2}+B^{2}+C\right) .
$$

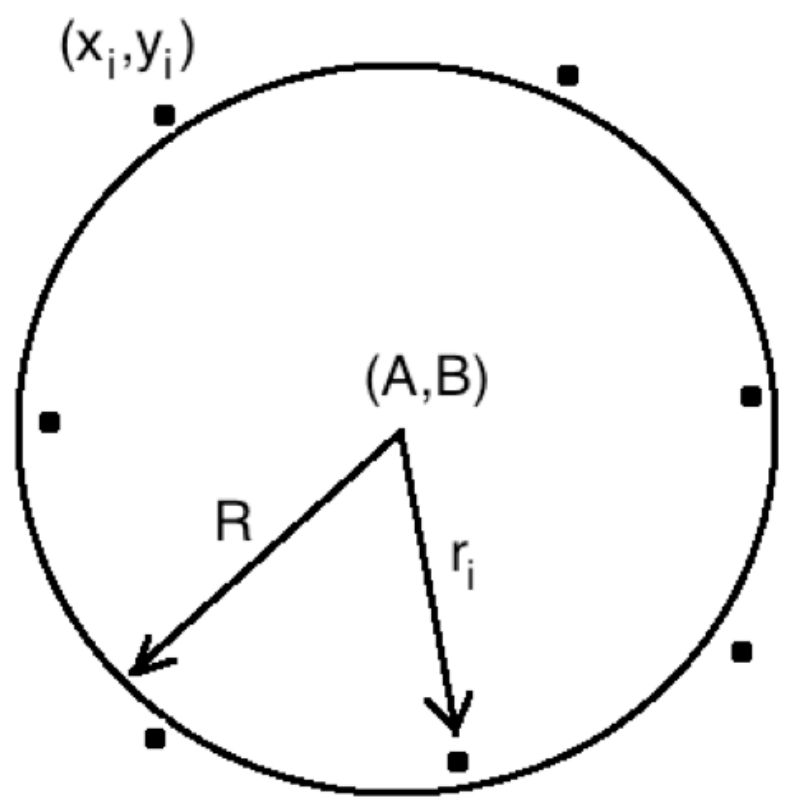

Figure 10. Circle fit schematic. 
The droplet edge coordinates are determined by considering the structure of the diffracted light zone at the droplet edge. For this we rescale the average intensities on either side of the diffraction zone such that the average intensity in the shadow zone is zero and the average intensity in the bright zone is unity. If we term this rescaled intensity $I_{n}$, then the droplet edge is located where $I_{n}=0.5$ if the incident light is purely incoherent and at $\mathrm{I}_{\mathrm{n}}=$ 0.25 if the incident light is purely coherent (Senchenko and Chugui, 2011; Fowler and Litorja, 2003). For partially coherent light, the droplet edge will be at a position where $I_{n}$ is between 0.25 and 0.5 (Senchenko and Chugui, 2011; Fowler and Litorja, 2003). An advantage of this method is that sub-pixel resolution of the droplet edge location is possible. The use of normalized intensities also allows for variations in background intensity around the droplet periphery. However, because background intensity fluctuations could make it difficult to determine the average intensity in the bright zone away from the droplet edge, it was assumed that the normalized peak intensity was the same everywhere around the droplet edge and average bright zone intensities were calculated using the peak intensity, which was generally easily observed, and the average intensity within a droplet, which typically had low noise levels.

A custom image processing code was written in C (Hanly and Koffman, 2010) and Python (Zelle, 2010) for the analysis. This code would scan the pixel intensity of each row and column of a region of interest that contained a droplet image. Candidate pixels for the droplet edge were identified based on $I_{n}$ and a circle was fit using the analysis outlined above. The k-means outlier detection algorithm (Jones et al., 2013) was then used to identify pixels that were not close to the circle edge - these pixels were rejected and a new circle was fit to the data. This procedure was repeated until no further outliers were detected.

Representative plots corresponding to this analysis are shown in Figure 11 and Figure 12. Figure 4a shows the digital image that was analyzed. Figure 11a shows a plot of the data set from the first iteration and Figure 11b and Figure 11c show plots for the second and third iterations.
The data in Figure 11 were calculated assuming that the normalized intensity that corresponds to the edge was $I_{n}=0.25$. Also in Figure 11 are histograms that show the distribution of the distance of the data points from the center of the best-fit circle - this distance is denoted as " $r$ " in Figure 11. The best-fit circle is shown as the red line. The diameters of the best-fit circles in Figures $11 \mathrm{abc}$ are $1.22 \mathrm{~mm}, 0.995 \mathrm{~mm}$, and 0.972 $\mathrm{mm}$, respectively. The standard deviations corresponding to the histograms in Figures $11 \mathrm{abc}$ are $0.213 \mathrm{~mm}, 0.048 \mathrm{~mm}$, and $0.009 \mathrm{~mm}$, respectively. The plots in Figure 11 clearly indicate that use of this outlier-detection procedure reduced the uncertainty in the edge location, which is also evident when the standard deviations in $r$ are compared.

Figure 12 shows the diameters that were calculated for the sphere in Figure 11a for different normalized intensities that were assumed to correspond to the edge. The normalized intensity that best corresponded to the edge was $I_{n}$ $=0.31$. This value of $\mathrm{I}_{\mathrm{n}}$ yielded a sphere diameter of $1.00 \mathrm{~mm}$, which is the same as the actual sphere diameter of $1.00 \mathrm{~mm}$ measured using a micrometer. The data in Figure 12 also show that it is important to correctly specify the edge $I_{n}$ value, as the diameters in Figure 12 range from $0.97 \mathrm{~mm}$ to $1.06 \mathrm{~mm}$ as $I_{n}$ is varied over the range 0.25 to 0.50 .

\section{CONCLUSIONS}

Backlit droplet images were evaluated for droplet combustion experiments that have been performed on the ISS. The focus of the present analyses was on non-sooting or lightly-sooting droplets and influences of diffraction, interference, and partial coherence on droplet images were considered via Fourier optics modeling. It was found that light diffraction at the droplet edge can contribute significantly to errors in droplet size measurements. Other error sources include background light variations and partial coherence effects. An image-processing algorithm was proposed to account for the effects of diffraction, partial coherence, and background light variations on measurements of droplet sizes. 


\section{Droplet Combustion Image Analysis}

A
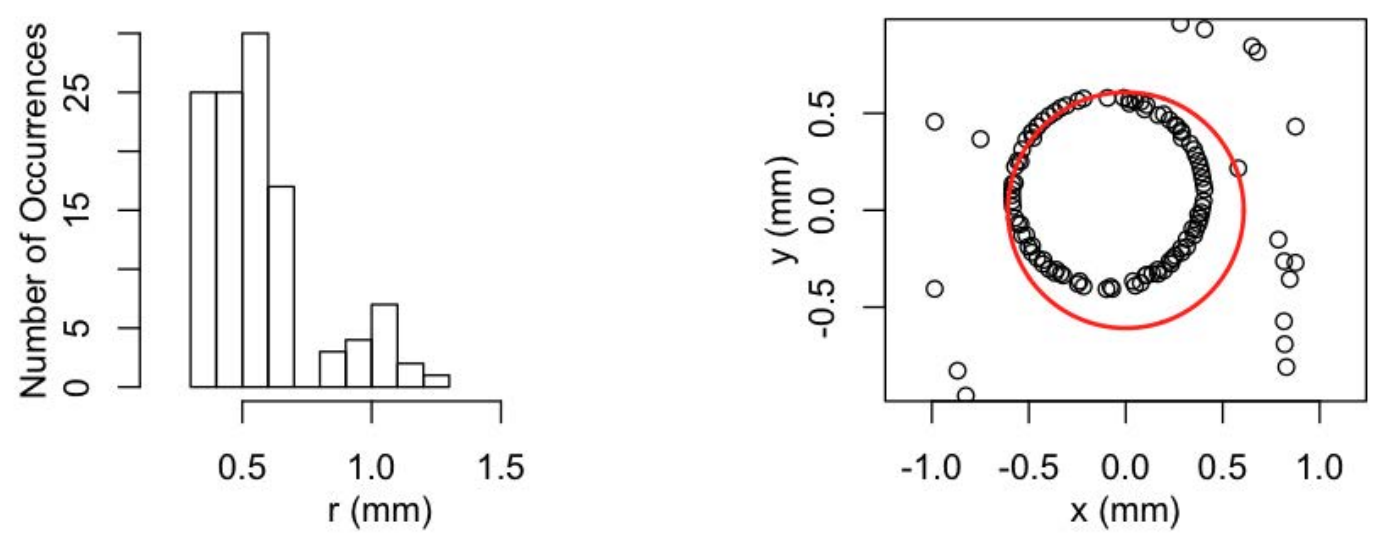

$\mathrm{B}$
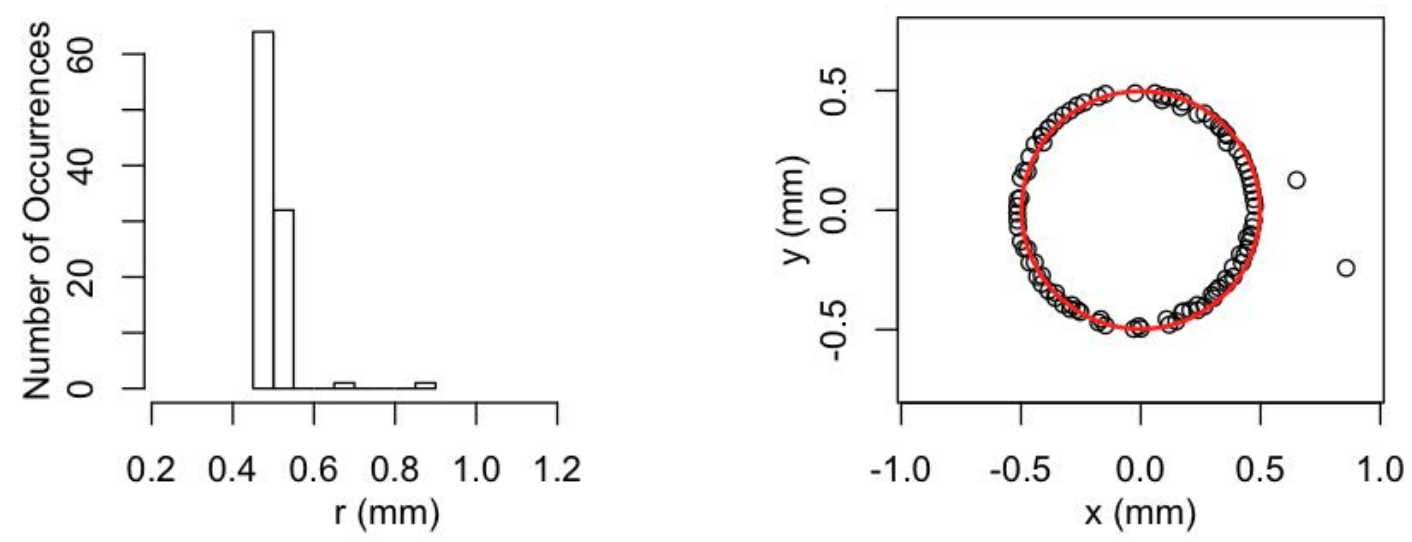

$\mathrm{C}$
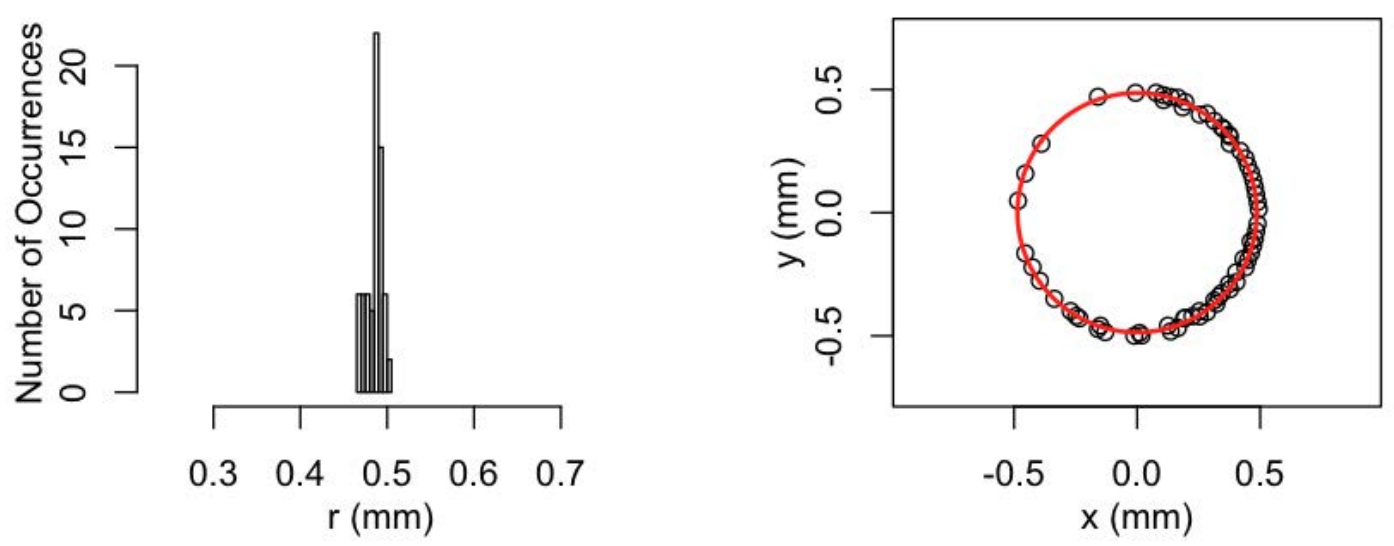

Figure 11. Results from an analysis of the image in Figure 4a: (a) first iteration; (b) second iteration; and (c) third iteration. 


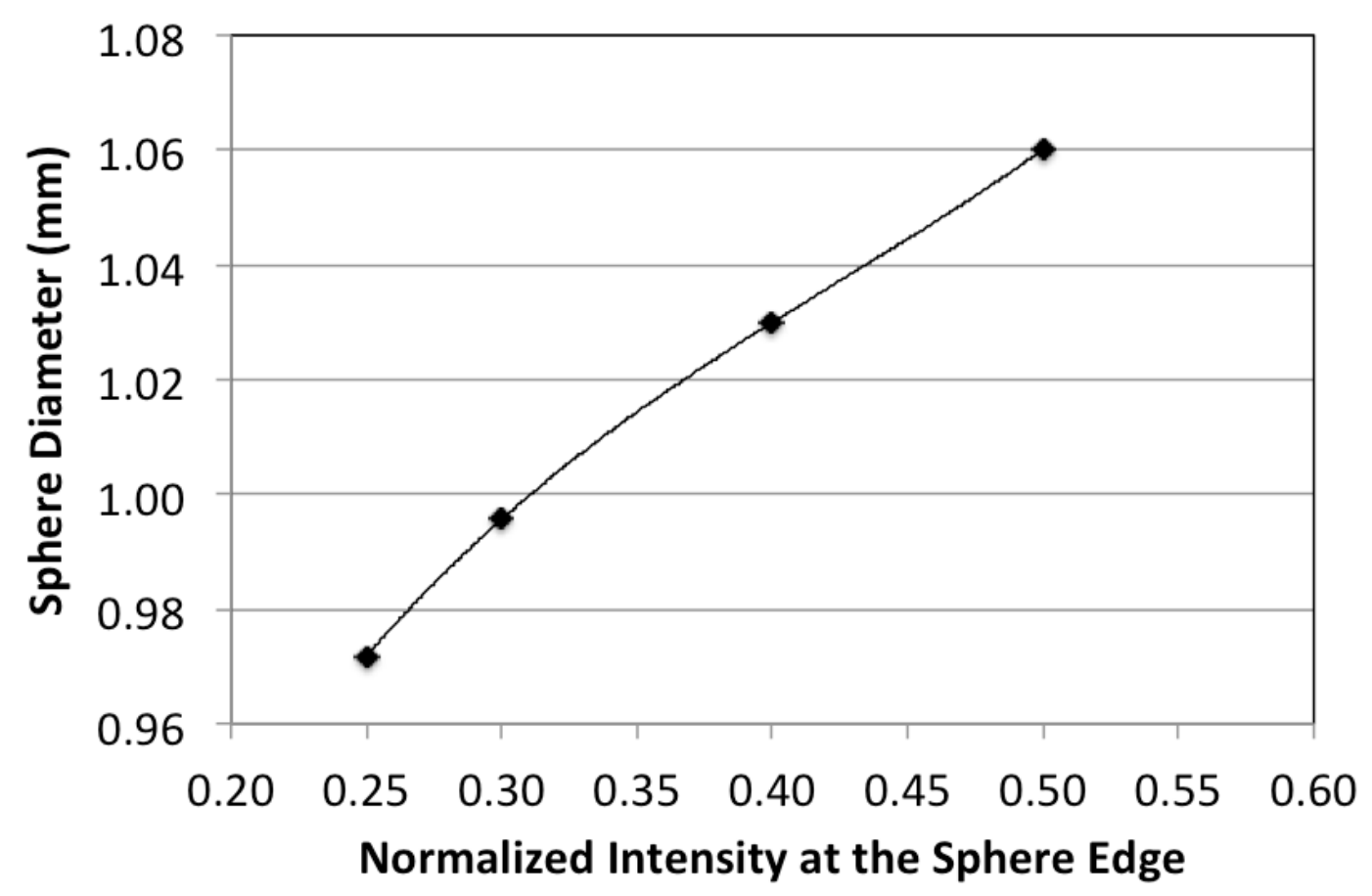

Figure 12. Calculated sphere diameter as a function of the normalized light intensity that was assumed to correspond to the sphere edge.

It would be useful to extend this research. For example, a Hough transform might be employed to rapidly determine approximate coordinates of the droplet edge, with further analyses then used to refine the droplet edge coordinates. It would also be of interest to consider the effects of strong sooting on diffraction, partial coherence, and determining the locations of droplet edges.

\section{ACKNOWLEDGEMENTS}

The financial support of NASA is gratefully acknowledged. The Technical Monitor was Dr. Daniel L. Dietrich. We appreciate discussions with V. Berg, D.L. Dietrich, F.L. Dryer, P. Ferkul, M. Hicks, V. Nayagam, and F.A. Williams. We also express our sincere gratitude to the management, engineering, and operations teams at NASA and Zin Technology, Inc., and the ISS astronauts who participated in the experiments.

\section{REFERENCES}

Dembia CL, Liu YC, Avedisian CT (2012) Automated data analysis for consecutive images from droplet combustion experiments. Image Analysis \& Stereology 31: $137-148$

Dietrich DL, Ferkul PV, Bryg VM, Nayagam N, Hicks MC, Williams FA, Dryer FL, Shaw BD, Choi MY, Avedisian CT (2013) Detailed Results from the Flame Extinguishment Experiment (FLEX) - March 2009 to December 2010. NASA/TP-2013216046, Cleveland, OH: NASA John H. Glenn Research Center

Fowler J, Litorja M (2003) Geometric area measurements of circular apertures for radiometry at NIST. Metrologia 40: S9-S12

Hanly J, Koffman E (2010) Problem Solving and Program Design In C, $\left(6^{\text {th }}\right.$ edn $)$. Boston: Addison-Wesley

Jones E, Oliphant T, Peterson P and others (20012014) SciPy: Open Source Scientific Tools for Python. Retrieved from http://www. scipy. org/

Kasa I (1976) A circle fitting procedure and its error analysis. IEEE Transactions on Instrumentation and Measurement 25: 8-14

Senchenko ES, Chugui YV (2011) Shadow inspection of $3 \mathrm{~d}$ objects in partially coherent 


\section{Droplet Combustion Image Analysis}

light. Measurement Science Review 11: 104107

Skinner TJ (1965) Energy Considerations, Propagation in Random Medium and Imaging in Scalar Coherence Theory. Ph.D. Thesis, Boston University

Voelz D (2011) Computational Fourier Optics: a MATLAB Tutorial. Bellingham, WA: SPIE
Webster JG (1999) The Measurement Instrumentation and Sensors Handbook. Boca Raton, FL: CRC Press

Zelle J (2010) Python Programming: an Introduction to Computer Science, (2nd edn). Wilsonville, OR: Franklin, Beedle \& Associates Inc. 